\title{
Evaluation of bowel preparation before colonoscopy by ultrasonographic monitoring of colonic fecal retention: a case series
}

\author{
Masaru Matsumoto1, Masayuki Fujioka², Toshihiko Okada 2,3, Yutaka Naka 2,3, Ayumi \\ Amemiya $^{4}$, Erina Matsushima ${ }^{4}$, Nao Tamai ${ }^{1,6}$, Yuka Miura ${ }^{1}$, Gojiro Nakagami, ${ }^{5,6}$ Hiromi \\ Sanada ${ }^{5,6}$
}

${ }^{1}$ Department of Imaging Nursing Science, Graduate School of Medicine, The University of Tokyo, Tokyo, ${ }^{2}$ Katsuragi Hospital, Osaka, ${ }^{3} 2^{\text {nd }}$ department of Internal medicine, Osaka Medical College, Osaka, ${ }^{4}$ Department of Nursing Physiology, Graduate School of Nursing, Chiba University, Chiba, ${ }^{5}$ Department of Gerontological Nursing / Wound Care Management, Graduate School of Medicine, The University of Tokyo, Tokyo, ${ }^{6}$ Global Nursing Research Center, Graduate School of Medicine, The University of Tokyo, Tokyo, Japan.

\begin{abstract}
Aims: While bowel preparation for colonoscopy is the key to successful examination, taking laxatives and showing stools to others causes both physical and mental distress to the patient. Thus, an alternative method to evaluation bowel preparation is necessary. In the current study, we studied the colonic fecal retention by ultrasonography (US) and examined the US finding which reflected completion of BP. Material and methods: The subjects were outpatients who underwent colonoscopy. This report summarizes the ultrasonographic images of patients who underwent multiple US examinations for all five sites of the colon just before, during, and immediately after bowel preparation. According to the standard protocol, the patients took 2 L of polyethylene glycol-ascorbic acid as a laxative, which was discontinued when the nurse visually judged the stool to be clear. Results: Seven patients in their $50 \mathrm{~s}-80$ s, none of whom were unable to complete a colonoscopy due to residual feces were included in study. Following bowel preparation, the US images showed anechoic areas with haustration in four or all five areas of the colon. Three of the seven patients received low-dose laxatives (1.1-1.2 L); all three had watery stools in three or more colon areas and none of them were constipated at the time of taking $1 \mathrm{~L}$ of laxatives. Conclusions: Completion of bowel preparation can be assessed by the observation of anechoic areas with haustration in multiple colonic sites by ultrasonography.

Keywords: ultrasonography; colonoscopy; fecal retention; laxative; bowel preparation
\end{abstract}

\section{Introduction}

Colonoscopy is the gold standard procedure for the diagnosis and treatment of colon diseases [1]. Bowel preparation (BP) is critical for high quality colonoscopy, as it is closely related to the quality of the procedure [2].

Received 26.08.2020 Accepted 12.12.2020

Med Ultrason

2021, Vol. 23, No 2, 147-152

Corresponding author: Hiromi Sanada, PhD, RN, WOCN, FAAN Global Nursing Research Center, Graduate School of Medicine, The University of Tokyo 7-3-1 Hongo, Bunkyo-ku, Tokyo, 113-0033, Japan

Phone: (+81) 3-5841-3419

Fax: (+81) 3-5841-3442

E-mail: hsanada-tky@umin.ac.jp
Previous studies have indicated that up to one-quarter of colonoscopies may be conducted with inadequate BP $[3,4]$, and suboptimal BP is thought to occur in up to $20 \%$ of all colonoscopy procedures $[5,6]$. Insufficient BP increases the requirement for repeat examinations, which ultimately increases healthcare costs and patient burden $[7,8]$. Therefore, it is necessary to be able to evaluate BP appropriately, perform bowel cleansing correctly and properly complete BP.

BP is also associated with patient distress. First, a nurse usually determines the degree of bowel cleansing from the color and properties of the stool discharged by a patient [9]. In other words, the patient's excretion is seen by others, which may cause mental distress to patients. Therefore, it is important to determine another method to directly visualize the condition of the colon and 
manage bowel cleansing. Second, patients who undergo colonoscopy usually have to take a considerable amount of laxatives, often around 2L [10], and taking more laxatives than is necessary may cause physical distress. The laxative requirement and BP before colonoscopy should depend on the individual's colonic condition, so as to minimize physical distress. Indeed, it has been reported that the quality of BP is different between patients with and without constipation [11]. Therefore, it is important to develop a visualization tool that can directly and non-invasively monitor the state of the patient's colon and manage BP.

Previous studies have reported that US can visualize fecal retention in the colon and some studies have shown that stool retention can be assessed by the presence of a crescent-shaped hyperechoic area [12-16]. The aim of our study was to evaluate by US the colonic fecal retention in patients prepared for colonoscopy and to establish which US findings reflect the completion of BP.

\section{Material and methods}

This study, conducted in May 2019, was approved by the Ethical Committee of the University of Tokyo (No. 2020017NI). The institutional review board approved this case series and waived the need for informed consent from patients; all participants were given the opportunity to opt-out by the poster and on the web site regarding the use of data.

\section{Participants and setting}

The study subjects were outpatients who underwent colonoscopy in a Japanese acute care hospital. In this hospital, patients with constipation or those who did not want to take large doses of laxatives were often checked for fecal retention using US during BP. A maximum of three US examinations were conducted (fig 1) and the BP was considered to be finished when the stool was judged to be transparent by a nurse using photographic examples of rectal effluent [9].

\section{Bowel preparation}

BP was standardized as follows: The day before the examination, intakes were restricted to a light, low-fiber breakfast and after midday, only clear liquids were allowed. In addition, the evening before the examination, patients were required to take three Sennoside tablets. On the day of the examination, patients were hospitalized in the outpatient unit for the second phase of colon preparation with $2 \mathrm{~L}$ polyethylene glycol-ascorbic acid (MoviPrep ${ }^{\circledR}$, EA Pharma Co.,Ltd., Tokyo, Japan). Nurses confirmed the stool color and properties in the toilet when the patient determined that the stool became a clear liquid.

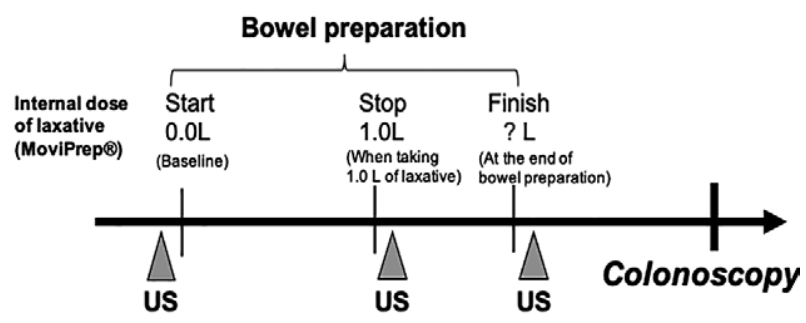

Fig 1. Ultrasonography during bowel preparation. A maximum of three ultrasonographies (US) were conducted. Bowel preparation was considered to be finished when the nurse confirmed a clear stool in the toilet; the total oral dose at that time was recorded.

\section{Ultrasound}

US of the rectum, ascending, transverse, descending and sigmoid colon was performed using ultrasound systems (Aplio 500 and Aplio a450systems, Canon Medical System Corporation, Otawara, Tochigi, Japan) with a curved-array probe $(1.5-6.0 \mathrm{MHz})$. The resulting images were supplemented by transverse and longitudinal sonographic scans. All sonographic procedures were performed by a certified sonographer specializing in the abdominal region, with 20 years of experience. The sonographer was blinded to the results of the assessment for stool color and properties.

With reference to previous studies [12,17], we defined the US levels of fecal retention as follows: a weak/ strong fecal retention finding was indicated by a marginally highly echoic colonic lumen and posterior echo behind the colon on transverse images and a flattened outer boundary wall and highly echoic colon wall on longitudinal images; a watery stool retention was indicated by an anechoic area on transverse images and an anechoic area with haustration on longitudinal images [18]. The US finding of multiple reflections was determined to be gas retention. The typical ultrasonographic findings related to fecal retention are shown in figure 2 and 3.

\section{Colonoscopy}

Several trained gastroenterologists performed the colonoscopies. A colonoscopy was defined as incomplete when there was no visualization of anatomic features, such as the ileocecal valve, appendiceal orifice, ileocolonic anastomosis, or terminal ileum, as previously described [19].

\section{Results}

A total of seven patients (five men, $71.4 \%$ ) were included in study and their characteristics are summarized in Table I. The patients' age at presentation ranged from 50 to 83 years, with a median age of 69 years. Six patients $(85.7 \%)$ took Sennoside $(12 \mathrm{mg} ; 3$ tablets after dinner 

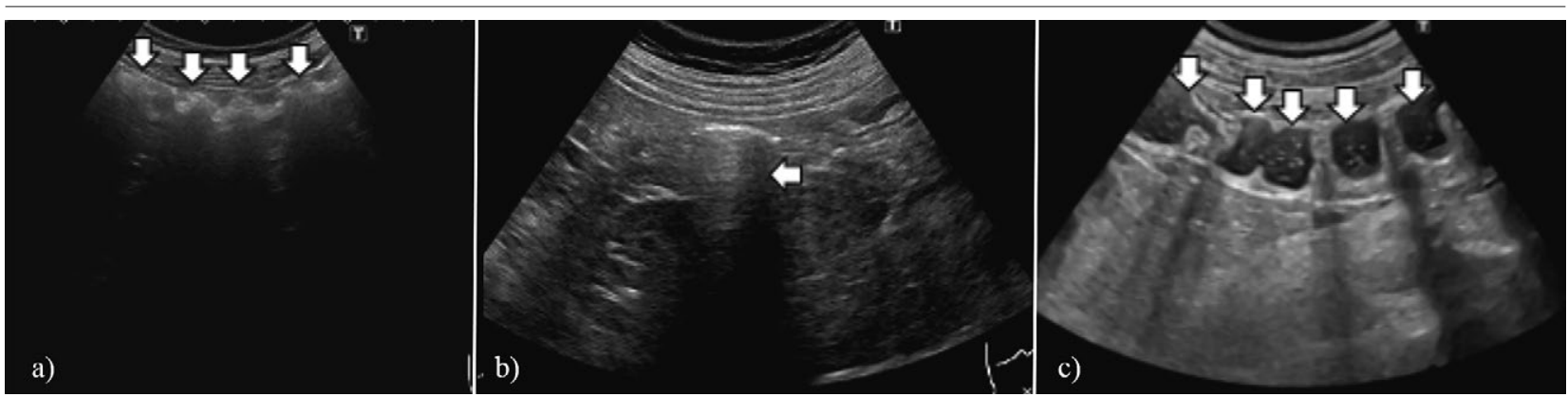

Fig 2. Ultrasonographic findings related to fecal retention: a) colonic fecal retention in a 69-year-old male patient. A longitudinal ultrasonographic image showing hyperechoic areas and acoustic shadow with haustrations on the wall of the descending colon (arrows); b) colonic gas in a 59-year-old male patient. A longitudinal ultrasonographic image showing multiple reflections on the wall of the descending colon (arrow); c) watery stool in a 83-year-old male patient. A longitudinal ultrasonographic image showing anechoic areas, indicating water retention in the descending colon (arrows).

and 3 tablets before sleep) prescribed by their doctors. Three patients $(42.9 \%)$ had daily bowel movements, with normal stool properties and amounts. On the morning of the colonoscopy, six of the seven patients $(85.7 \%)$ had a bowel movement. Time from the start of $2 \mathrm{~L}$ polyethylene glycol-ascorbic acid to taking $1 \mathrm{~L}$ ranged 67 to 80 minutes, with a median time of 73 minutes. Time from the start of $2 \mathrm{~L}$ polyethylene glycol-ascorbic acid to the completion of bowl preparation ranged 91 to $155 \mathrm{~min}$ utes, with a median time of 121 minutes.

The change in ultrasonographic findings, total dose of laxative and degree of bowel cleanliness are shown in Table II. None of the patients were determined to have

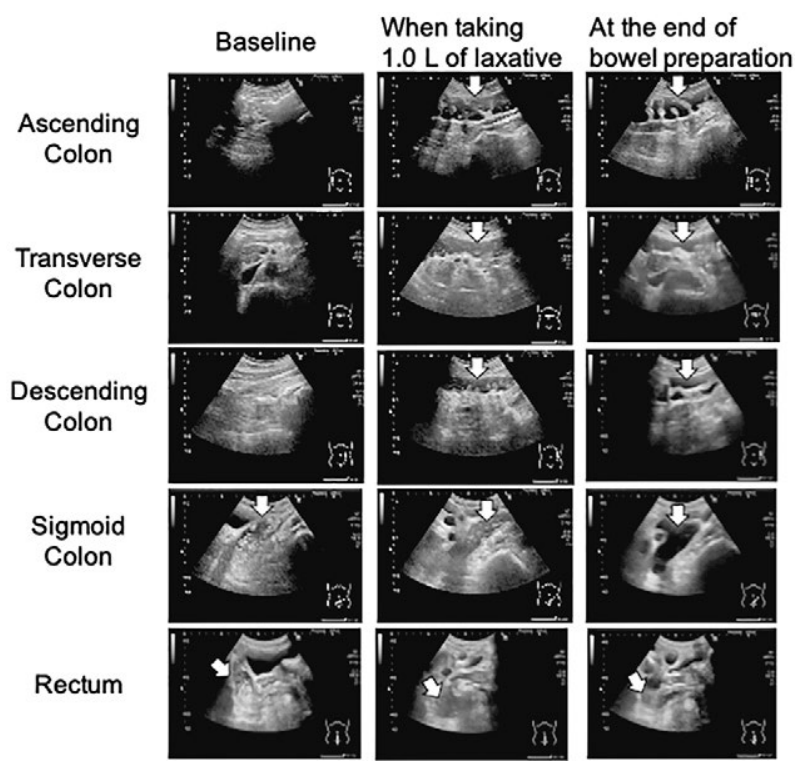

Fig 3. Time course of ultrasonographic images at colon in a 69-year-old male patient (ID1). Longitudinal ultrasonographic images showing anechoic areas, indicating water retention in the descending colon (arrows). The stool was judged to be transparent by the nurse, and the patient finished bowel preparation after taking $1.1 \mathrm{~L}$ of laxative. an incomplete colonoscopy. The ultrasound images at the completion of BP showed anechoic areas with haustration in four, or all five areas of the colon. Three patients (Cases 1-3) received low-dose laxatives (1.1 to $1.2 \mathrm{~L}$ ); the US images of these three patients showed anechoic findings indicating watery stool retention in three or more colon areas. None of the three patients were constipated at the time of consuming $1 \mathrm{~L}$ of laxatives.

\section{Discussions}

This study firstly showed colonic US imaging at BP before colonoscopy. The US results demonstrated that four or all five areas of the colon showed anechoic areas with haustration and the lack of hyperechoic area in the stool indicated that BP had been completed. Patients who received $1 \mathrm{~L}$ of laxative, had watery stools in three or more of the colon sites and no fecal retention in any of these sites, received a smaller dose of laxatives. Therefore, it is possible to determine by US that BP has been completed.

The US images at the completion of BP showed anechoic areas with haustration in four or all five areas of the colon, which indicated that BP was complete. As a practical point for the future, endoscopy nurses should be able to observe the colon with a hand-held US device to assess BP completion without causing the patient the mental distress of having to have their stool observed. In the same way, that the effectiveness of hand-held US observation and treatment of constipation has been shown $[20,21]$, the effectiveness of US observation by endoscopy nurses needs to be studied in the near future. There will also be a need to develop a US education program for nurses to observe colonic fecal retention [22]. In addition, non-invasive assessment of BP with US may help to determine the timing of BP completion for each patient and minimize the physical distress caused by laxatives. Indeed, constipated patients have been reported 
Med Ultrason 2021; 23(2): 147-152

Table II. Change of colonic contents based on ultrasonographic findings, total dose of laxative and degree of bowel cleanliness.

\begin{tabular}{|c|c|c|c|c|c|}
\hline \multirow[t]{2}{*}{ ID } & \multirow[t]{2}{*}{ Site } & \multicolumn{3}{|c|}{ Ultrasonographic findings } & \multirow{2}{*}{$\begin{array}{l}\text { Total dose of } \\
\text { laxative }\end{array}$} \\
\hline & & Baseline & When taking $1 \mathrm{~L}$ of laxative & At the end of bowel preparation & \\
\hline \multirow[t]{5}{*}{1} & $\mathrm{AC}$ & + & watery stool & watery stool & $1.1 \mathrm{~L}$ \\
\hline & $\mathrm{TC}$ & + & watery stool & watery stool & \\
\hline & $\mathrm{DC}$ & - & watery stool & watery stool & \\
\hline & $\mathrm{SC}$ & watery stool & watery stool & watery stool & \\
\hline & $\mathrm{R}$ & watery stool & watery stool & watery stool & \\
\hline \multirow[t]{5}{*}{2} & $\mathrm{AC}$ & + & watery stool & watery stool & $1.2 \mathrm{~L}$ \\
\hline & $\mathrm{TC}$ & + & watery stool & watery stool & \\
\hline & $\mathrm{DC}$ & - & watery stool & watery stool & \\
\hline & $\mathrm{SC}$ & - & watery stool & watery stool & \\
\hline & $\mathrm{R}$ & - & - & watery stool & \\
\hline \multirow[t]{5}{*}{3} & $\mathrm{AC}$ & - & watery stool & watery stool & $1.2 \mathrm{~L}$ \\
\hline & $\mathrm{TC}$ & - & watery stool & watery stool & \\
\hline & $\mathrm{DC}$ & - & watery stool & watery stool & \\
\hline & $\mathrm{SC}$ & gas & - & watery stool & \\
\hline & $\mathrm{R}$ & + & - & - & \\
\hline \multirow[t]{5}{*}{4} & $\mathrm{AC}$ & + & + & watery stool & $1.4 \mathrm{~L}$ \\
\hline & $\mathrm{TC}$ & - & watery stool & watery stool & \\
\hline & $\mathrm{DC}$ & - & watery stool & watery stool & \\
\hline & $\mathrm{SC}$ & - & watery stool & watery stool & \\
\hline & $\mathrm{R}$ & - & - & watery stool & \\
\hline \multirow[t]{5}{*}{5} & $\mathrm{AC}$ & watery stool & watery stool & watery stool & $1.5 \mathrm{~L}$ \\
\hline & $\mathrm{TC}$ & watery stool & watery stool & watery stool & \\
\hline & $\mathrm{DC}$ & + & watery stool & watery stool & \\
\hline & $\mathrm{SC}$ & gas & watery stool & watery stool & \\
\hline & $\mathrm{R}$ & - & + & watery stool & \\
\hline \multirow[t]{5}{*}{6} & $\mathrm{AC}$ & watery stool & watery stool & watery stool & $1.6 \mathrm{~L}$ \\
\hline & $\mathrm{TC}$ & - & watery stool & watery stool & \\
\hline & $\mathrm{DC}$ & gas & - & watery stool & \\
\hline & $\mathrm{SC}$ & + & gas & watery stool & \\
\hline & $\mathrm{R}$ & - & - & watery stool & \\
\hline \multirow[t]{5}{*}{7} & $\mathrm{AC}$ & + & no data & watery stool & $2.0 \mathrm{~L}$ \\
\hline & $\mathrm{TC}$ & + & no data & watery stool & \\
\hline & $\mathrm{DC}$ & + & no data & watery stool & \\
\hline & $\mathrm{SC}$ & + & no data & watery stool & \\
\hline & $\mathrm{R}$ & - & no data & - & \\
\hline
\end{tabular}

AC: Ascending colon, TC: Transverse colon, DC: Descending colon, SC: Sigmoid colon, R: Rectum, +: Hyperechoic finding indicating fecal retention, -: No specific finding

to have less effective BP than non-constipated patients $[11,18]$ and in these cases, observation-based, individualized support for BP is required before colonoscopy. In the cases IDs 1, 2 and 3, all received low doses of laxatives $(1.1-1.2 \mathrm{~L})$; these three patients had watery stools in three or more colonic areas and no fecal retention was observed in any of these cases after $1 \mathrm{~L}$ of laxative. ID7 usually defecated twice a week, which was the least frequent of the participants in this study. Although data are not available for $1 \mathrm{~L}$ of laxatives, patients with constipation may need to take more laxatives than those without symptoms. It will be necessary to investigate whether evaluation of BP using US can reduce the amount of laxatives taken by patients.

The present study has some limitations. Because this study was based on the assumption that polyethylene glycol was taken internally, we did not consider the use of sodium phosphate or sodium picosulfate with magnesium citrate. Furthermore, the quality of BP was not assessed from endoscopic images [23]. Future studies will need to investigate the relationship between US images and endoscopic images. 
Conclusions. Our results demonstrate that completion of BP can be assessed by observing the anechoic area with haustration observed in multiple colonic sites by US.

Acknowledgments: The authors are deeply grateful to the study participants, all of whom contributed greatly to this study. This research was supported by joint research funding with the Department of Imaging Nursing Science.

Conflict of interest: Masaru Matsumoto, Nao Tamai and Yuka Miura belong to a social collaboration department that receives funding from Fujifilm Corporation.

\section{References}

1. Mihalko SL. Implementation of colonoscopy for mass screening for colon cancer and colonic polyps: efficiency with high quality of care. Gastroenterol Clin North Am 2008;37:117-128.

2. Coriat R, Polin V, Oudjit A, et al. Gastric emptying evaluation by ultrasound prior colonoscopy: an easy tool following bowel preparation. World J Gastroenterol 2014;20:1359113598.

3. Harewood GC, Sharma VK, de Garmo P. Impact of colonoscopy preparation quality on detection of suspected colonic neoplasia. Gastrointest Endosc 2003;58:76-79.

4. Lebwohl B, Kastrinos F, Glick M, Rosenbaum AJ, Wang T, Neugut AI. The impact of suboptimal bowel preparation on adenoma miss rates and the factors associated with early repeat colonoscopy. Gastrointest Endosc 2011;73:1207-1214.

5. Cheng CL, Liu NJ, Tang JH, et al. Predictors of Suboptimal Bowel Preparation Using 3-1 of Polyethylene Glycol for an Outpatient Colonoscopy: A Prospective Observational Study. Dig Dis Sci 2017;62:345-351.

6. Radaelli F, Paggi S, Repici A, et al. Barriers against splitdose bowel preparation for colonoscopy. Gut 2017;66:14281433.

7. Rex DK, Imperiale TF, Latinovich DR, Bratcher LL. Impact of bowel preparation on efficiency and cost of colonoscopy. Am J Gastroenterol 2002;97:1696-1700.

8. Clark BT, Rustagi T, Laine L. What level of bowel prep quality requires early repeat colonoscopy: systematic review and meta-analysis of the impact of preparation quality on adenoma detection rate. Am J Gastroenterol 2014;109:1714-1723.

9. So H, Boo SJ, Seo H, et al. Patient descriptions of rectal effluents may help to predict the quality of bowel preparation with photographic examples. Intest Res 2015;13:153-159.

10. Khan MA, Patel KB, Nooruddin M, et al. Polyethylene Glycol-3350 (Miralax $\left.{ }^{\circledR}\right)+1.9$-L sports drink (Gatorade $\left.{ }^{\circledR}\right)+2$ tablets of bisacodyl results in inferior bowel preparation for colonoscopy compared with Polyethylene Glycol-Ascorbic Acid (MoviPrep ${ }^{\circledR}$ ). Turk J Gastroenterol 2018;29:67-74.

11. Kunz L, Gillespie D. A Comparison of Bowel Preparations for Colonoscopy in Constipated Adults. Gastroenterol Nurs 2017;40:364-372.

12. Matsumoto M, Tanaka S, Yabunaka K, et al. Ultrasonographic evaluation of changes over time in one defecation cycle in adults with functional constipation: A report of two cases. Drug Discover Ther 2018;12:304-308.

13. Yabunaka K, Matsumoto M, Yoshida M, et al. Assessment of rectal feces storage condition by a point-of-care pocketsize ultrasound device for healthy adult subjects: A preliminary study. Drug Discov Ther 2018;12:42-46.

14. Yabunaka K, Matsuo J, Hara A, et al. Sonographic visualization of fecal loading in adults comparison with computed tomography. J Diagn Med Sonog 2015;31:86-92.

15. Yabunaka K, Nakagami G, Komagata K, Sanada H. Ultrasonographic follow-up of functional chronic constipation in adults: A report of two cases. SAGE Open Med Case Rep 2017;5:2050313x17694234.

16. Matsumoto M, Tsutaoka T, Nakagami G, et al. Deep learning-based classification of rectal fecal retention and analysis of fecal properties using ultrasound images in older adult patients. Jpn J Nurs Sci 2020;17:e12340.

17. Kawamura T, Nishiguchi T. Congenital Chloride Diarrhea (CCD): A Case Report of CCD Suspected by Prenatal U1trasonography and Magnetic Resonance Imaging (MRI). Am J Case Rep 2017;18:707-713.

18. Fan XP, Zhu Q, Zhou YJ, Ma T, Xia CX, Huang HL. Comparative Study of Three Regimens of Bowel Preparation Before Transabdominal Ultrasonography of the Colon. U1trasound Med Biol 2016;42:2140-2145.

19. Neerincx M, Terhaar sive Droste JS, Mulder CJ, et al. Colonic work-up after incomplete colonoscopy: significant new findings during follow-up. Endoscopy 2010;42:730735 .

20. Matsumoto M, Yabunaka K, Yoshida M, et al. Improvement of Constipation Symptoms in an Older Adult Patient by Defecation Care Based on Using a Handheld Ultrasound Device in Home Care Settings: A Case Report. J Wound Ostomy Continence Nurs 2020;47:75-78.

21. Matsumoto M, Yoshida M, Yabunaka K, et al. Safety and efficacy of a defecation care algorithm based on ultrasonographic bowel observation in Japanese home-care settings: a single-case, multiple-baseline study. Geriatr Gerontol Int 2020;20:187-194.

22. Matsumoto M, Yoshida M, Miura Y, et al. Feasibility of the constipation point-of-care ultrasound educational program in observing fecal retention in the colorectum: a descriptive study. Jpn J Nurs Sci 2020. doi:10.1111/jjns.12385.

23. Kastenberg D, Bertiger G, Brogadir S. Bowel preparation quality scales for colonoscopy. World J Gastroenterol 2018;24:2833-2843. 